\title{
EXTENSION UNIVERSITARIA Y TIC. REFLEXIONES DE LA PRÁCTICA DOCENTE EN LA PROBLEMÁTICA DE LA EDUCACIÓN PATRIMONIAL
}

\section{(UNIVERSITY EXTENSION AND ITC. REFLECTIONS OF THE EDUCATIONAL PRACTICE IN THE PROBLEMATIC ONE OF THE PATRIMONIAL HERITAGE)}

Mariela Eleonora Zabala

María Isabel Roura Galtés

Susana Beatriz Assandri

Universidad Nacional de Córdoba (Argentina)

\section{RESUMEN}

El objetivo de este artículo es reflexionar acerca de las posibilidades que brindan las nuevas Tecnologías de Información y Comunicación (TIC) para el desarrollo de programas educativos de Extensión Universitaria.

Las prácticas docentes que sustentan este trabajo abordan temas de Educación Patrimonial desarrolladas en el Programa: "Educación y Museos. Patrimonio para todos", Facultad de Filosofía y Humanidades, Universidad Nacional de Córdoba, Argentina, creado para ser dictado íntegramente en la modalidad de educación virtual. A diferencia de la oferta formativa que ofrecen la mayoría de las Universidades dentro de la virtualidad, referidas casi exclusivamente a la educación superior de grado y postgrado, la oferta que se analiza en este artículo es abierta al público en general.

Palabras clave: extensión universitaria, educación patrimonial, museo universitario, tecnologías de información y comunicación, TIC, educación virtual.

\begin{abstract}
The aim at this article is to reflect about the possibilities that offer the new Information and Communication Technologies (ICT) for the development of educative programs of University Extension.

The educational practices that sustain this work approach subjects of Heritage Education within the modality of virtual education, and they have been carried out within the framework of the Program: "Education and Museums. Heritage for all of us", Faculty of Philosophy and Humanities, National University of Córdoba, Argentina.
\end{abstract}


Unlike the formative supply which offer most of the Universities within the virtuality, referred almost exclusively to the superior education of degree and postdegree, the supply that is analyzed in this article is opened to the public in general.

Keywords: university extension, educational heritage, university museum, information and comunication technologies, ICT, virtual education.

En este artículo se analiza la importancia de la incorporación de las Tecnologías de Información y Comunicación (TIC) en actividades de Extensión Universitaria. El análisis está basado en la experiencia docente en el marco del "Programa Educación y Museos, Patrimonio para Todos"1 de la Secretaría de Extensión de la Facultad de Filosofía y Humanidades de la Universidad Nacional de Córdoba, en coparticipación con el Museo de Antropología, dependiente de la mencionada Facultad, y el "Museo Casa del Virrey Liniers y Estancia Jesuítica”, Alta Gracia, Córdoba (dependiente de la Dirección Nacional de Patrimonio y Museos Secretaría de Cultura de la Presidencia de la Nación- Museo de Gestión Nacional). Este es uno de los programas que la Secretaría de Extensión ofrece dentro de la modalidad educativa on line, basada en el uso de la plataforma virtual Moodle².

Comenzaremos por analizar brevemente el rol de los Museos Universitarios dentro de las políticas de Extensión Universitaria y cómo se llevan a cabo estas ideas mediante talleres y cursos presenciales sobre Educación Patrimonial (en adelante EP), para luego arribar al análisis de lo que significó la incorporación de las nuevas tecnologías de información y comunicación (en adelante TIC) en nuestra metodología de trabajo.

\section{MUSEOS UNIVERSITARIOS Y EXTENSIÓN UNIVERSITARIA}

Los museos son espacios socialmente legales y legítimos para seleccionar, investigar, conservar y difundir los estudios sobre el patrimonio que custodian. A nivel mundial los museos universitarios poseen un Comité diferenciado en el International Council of Museums ${ }^{3}$ (ICOM), como es el Comité Internacional de Museos Universitarios, porque se les reconoce que tienen un tipo de gestión peculiar por depender del presupuesto universitario, por el recurso humano especializado en la disciplina académica que conforman los equipos de trabajo, por los proyectos de investigación multidisciplinares, por la realización de actividades culturales multidisciplinares y por el modo en que se construyen sus colecciones. Las colecciones de los museos universitarios se conforman, fundamentalmente, a partir de estudios 
y trabajos de investigación, de donaciones de particulares y, antiguamente, por compra. La peculiaridad de estos museos reside en que la investigación da origen a la formación de la colección, en su mayoría (Assandri y Zabala, 2002).

El Museo de Antropología de la Facultad de Filosofía y Humanidades de la Universidad NacionaldeCórdoba,Argentina, comoinstitución universitariaalservicio de la sociedad y su desarrollo, tiene entre sus objetivos proponer nuevas metodologías pedagógicas que permitan extender el ámbito cultural de la Universidad hacia otros sectores de la sociedad, y dar respuesta a las frecuentes demandas de formación sobre temas patrimoniales que realizan municipios, comunas, museos, centros culturales, escuelas y agrupaciones étnicas. Entre las inquietudes de los demandantes podemos señalar la preocupación por la pérdida de bienes patrimoniales debido a la falta de políticas estatales, preocupación por los cambios en el ambiente que afectan la conservación de bienes culturales, la búsqueda de recuperación y revaloración de sus bienes patrimoniales y la necesidad de registrar su patrimonio.

\section{EDUCACIÓN PATRIMONIAL EN EL MARCO DE LA EXTENSIÓN UNIVERSITARIA}

La EP es una estrategia útil para promover el diálogo entre la Universidad y la Sociedad, ya que su objetivo principal consiste en despertar la inquietud por conocer el patrimonio, no sólo en los especialistas, sino en todos los ciudadanos.

La extensión universitaria ofrece oportunidades de formación para un sector que no ha tenido posibilidades de acceder a una educación sistemática en el nivel superior. Teniendo en cuenta que en la provincia de Córdoba, Argentina, no contamos con una carrera Terciaria, ni Universitaria de Museología es muy importante la formación que se brinda desde este programa de extensión para los trabajadores de museos y centros patrimoniales. En este caso la extensión universitaria se ve facilitada por la aplicación de la modalidad virtual, ya que los posibles alumnos, adultos en su mayoría, tienen dificultades para la modalidad presencial por razones de trabajo, distancia o económicas.

El patrimonio ha tenido, y tiene, en las distintas sociedades un papel importante como legitimador de las acciones políticas, culturales y sociales. Los bienes culturales son patrimonio desde el momento en que un grupo humano los selecciona porque reconoce en ellos valores identitarios y/o testimoniales, y de este modo son legitimados por el grupo social que les otorga significado. Al atribuirle valores se 
determina qué bienes pasan a formar parte de los procesos de investigación y gestión de ese patrimonio, de manera que, de la valoración depende, en primera instancia, qué patrimonio es estudiado y cuál es relegado a un segundo plano o, incluso, al anonimato social, económico, político o científico. Es así que la estrecha relación existente entre el patrimonio, las prácticas propias de la vida de las comunidades y la investigación universitarias le confieren a aquel su carácter dinámico e identitario.

Por ello es necesario sustentar las actividades de EP a partir de una tarea permanente de análisis sobre los significados que se le atribuyen al patrimonio como soporte de la memoria colectiva.

\section{EDUCACIÓN PATRIMONIAL EN CONTEXTOS VIRTUALES}

Trabajar desde un entorno virtual la problemática patrimonial significa, desde el momento mismo de la planificación, sortear múltiples desafíos profesionales de orden pedagógico y conceptual.

La sola incorporación de las TIC viene a alterar la forma de organizar el pensamiento desde las nuevas posibilidades de acceso a la información y, sobre todo, propone una modalidad de aprendizaje en donde el sujeto que aprende es quien organiza su propio proceso educativo, en tiempos y espacios que él mismo determina (en un cyber, en su casa, en la oficina, en el mismo lugar o en distintos lugares cada día) con la única constante: el acceso a un aula virtual y la información que ésta brinda. Dentro de este nuevo entorno pedagógico, ¿̇cómo es posible educar en patrimonio en un contexto a-temporal y a-espacial como Internet?

En un contexto espacial parece clara la diferencia entre lo local y lo universal, entre lo cercano y lo lejano; sin embargo, en un entorno virtual y sin fronteras, como definimos a Internet, estos límites se vuelven mucho más complejos y, en cierta medida, carentes de sentido. Si pensamos en la EP, donde esta distinción resulta ser un elemento conceptual clave, nos encontramos, aparentemente, sin conexiones posibles entre Internet y la EP. Sin embargo, la mayor riqueza que puede ofrecer el entorno virtual es la posibilidad de que personas de diferentes espacios sociales y geográficos puedan interactuar relacionando conceptos clave con sus realidades culturales. Las comunidades virtuales dejan atrás las barreras de tiempo y espacio. Los docentes y los alumnos pueden no compartir un espacio físico o un tiempo determinado, pero la comunidad funciona igualmente en el ciberespacio. 
Este reconocimiento permite abordar un concepto clave para los estudios patrimoniales: la multiculturalidad. Internet es un espacio de todos los pronunciamientos, todos los sitios y todas las voces. Esto contribuye a construir aulas virtuales y foros que incorporan las diferencias, asumen las historias, las lenguas y culturas particulares de los sujetos del proceso educativo, abriéndose a la pluralidad. A partir de entonces es posible construir y resignificar conocimientos en torno al patrimonio, que permitan a las personas reconocer, valorar y posicionarse críticamente en relación con el patrimonio, tanto local como mundial.

Otro desafío tiene quever con la complejidad de los contenidos a abordar. Mientras en la educación tradicional el libro organiza en forma lineal la información, lo que permite que todos los alumnos trabajen sincrónicamente sobre los mismos temas, las TIC promueven otra forma de incorporar conocimientos, a partir de procesos de aprendizaje no lineales y de diálogos asincrónicos sobre alguna problemática común. Pero no alcanza con usar las TIC para poder hablar de aprendizaje virtual, es necesario ser capaces de pensar, de usarla creativamente y de manera productiva. Este es otro aprendizaje que se debe construir en forma simultánea a la incorporación de conocimientos sobre patrimonio. Tomando en cuenta que los únicos requisitos que plantea la Secretaría de Extensión para la admisión de participantes es que tengan estudios secundarios completos, posibilidad de conexión a internet y conocimientos mínimos sobre el uso de esta tecnología, ¿̇qué contenidos es posible abordar para trabajar con grupos tan heterogéneos? Un grupo destinatario que se compone de alumnos cuya formación de grado es diversa, cuyas edades también son dispares y que, además, deben interactuar desde muy distintos espacios geográficos nos desafía a pensar actividades didácticas muy distintas a las que se pueden plantear en espacios presenciales. Para que cada alumno pueda reelaborar los conceptos en su propia realidad espacio-temporal y a su vez pueda intercambiar experiencias y conocimientos con los demás participantes, es necesario proponer los canales adecuados que orienten los procesos de aprendizaje de los participantes sin limitar su creatividad y autonomía.

Es decir, la educación virtual se manifiesta como un nuevo modelo de enseñanzaaprendizaje, un escenario que permite una educación distinta a la educación presencial, promoviendo tanto en los alumnos como en los docentes: la construcción de nuevos modos de organizar y acceder a la información y los conocimientos; autonomía en uso de las nuevas tecnologías; el conocimiento de nuevos formatos en que se codifica la información: los formatos multimedia e hipertextuales; la modificación de vínculos con el conocimiento ya sea a través de procesos de simulación, de alteración, de producción. Un modelo no direccional, en el cual el 
docente no sólo entrega información sino que actúa como monitor u orientador, dando lugar a un aprendizaje donde todos aprenden en comunidad.

A partir de reconocer las potencialidades de este nuevo contexto, vincular los planteamientos conceptuales sobre patrimonio y nuevas tecnologías permite plantear estrategias destinadas a promover en los alumnos una comprensión de los conceptos abordados vinculándolos con el análisis de su realidad inmediata. Los participantes, al permanecer en su propio entorno profesional y social mientras desarrollan su proceso formativo, deben ser capaces de encontrar precisamente en ese ámbito los objetos de estudio para responder a las consignas que se plantean en las actividades. Este es el punto de partida que guía la propuesta de EP objeto de esta comunicación, y cuya estructura organizativa vincula instancias de aprendizaje y de comunicación para posibilitar la transmisión y la construcción de conocimientos (Assandri, Roura y Zabala, 2007).

\section{EL DESARROLLO DE LAS EXPERIENCIAS}

El Programa "Educación y Museos. Patrimonio para todos" fue creado para ser dictado a través de la modalidad virtual. Por lo tanto, todos los cursos que incluye, este Programa, fueron especialmente diseñados para adaptarse a esta modalidad.

El Área de Tecnología Educativa, de la Facultad de Filosofía y Humanidades, asesoró a los docentes para la elaboración de las propuestas de enseñanza de cada curso que se encaminaron en dos sentidos: el diseño de las aulas virtuales y de sus materiales de estudios. Esto conjugó las necesidades de capacitación de los docentes en el lenguaje y lógicas digitales, la exploración del campus, la toma de diferentes decisiones a partir del conocimiento y dominio del entorno de enseñanza por cada equipo docente, lo que dio lugar a ir creando, progresivamente, un estilo de enseñanza y de comunicación pedagógica con los participantes.

La estructura organizativa que se utiliza para llevar a cabo el Programa en el que se incluyen estos cursos, se basa en el uso de un Aula Virtual habilitada, como se dijo anteriormente, bajo la plataforma Moodle. A través del aula virtual, alumnos y profesores pueden acceder a materiales de clases, publicaciones, cronogramas y espacios de comunicación. El uso de Aulas Virtuales favorece el surgimiento (unas veces espontáneo y otras organizado) de redes, concebidas como ámbitos para el intercambio de conocimientos, propuestas, evaluaciones, etc., que favorecen los contactos entre profesionales más allá del momento en que se lleva a cabo el proceso 
educativo. Estas aulas constituyen, en sí mismas, un elemento de sustentabilidad, en la medida en que estimulan el aprendizaje permanente y son un incentivo para los intercambios entre quienes poseen intereses comunes.

La plataforma utilizada para el aula virtual consta de herramientas tecnológicas que pueden agruparse en recursos y actividades. Entre los recursos que se ofrecen para el desarrollo de los temas se pueden utilizar: páginas web, enlaces con otras páginas, bibliografía en formatos electrónicos, videos, presentaciones en power point, etc. La mayoría de estos recursos son de fácil acceso para los participantes y de mucha utilidad didáctica. Entre las actividades se proponen foros, tareas, chat, wiki4, glosarios, cuestionarios, etc. El uso que cada alumno hace de los recursos brindados es diferente y personalizado, y por ello es importante que las actividades planificadas por los docentes sean lo suficientemente claras y flexibles como para permitir que cada alumno pueda realizarlas partiendo de procesos diferentes.

Se puede elegir entre varios formatos de curso tales como una propuesta de duración periódica, ya sea semanal o quincenal, por temas o el formato social, basado en debates. En las experiencias que estamos analizando se han elegido formatos semanales o quincenales, es decir, se han ajustado los temas a abordar dentro de módulos que responden a períodos fijos de tiempo. Esto es sumamente importante en las propuestas virtuales, ya que permite a los alumnos y docentes una cierta constancia en el proceso de aprendizaje.

En correspondencia con cada módulo de aprendizaje se proponen foros en donde, a partir de una actividad se debaten conceptos como: memoria, identidad, arqueología, paisaje, procesos de patrimonialización, difusión del patrimonio. El foro electrónico es una de las herramientas tecnológicas más utilizadas, ya que favorece la interacción a distancia y asincrónica, y permite la discusión entre diferentes personas sobre un tema particular. Este uso del foro admite una instancia distinta de aprendizaje que puede inscribirse en lo que se denomina "aprendizaje colaborativo", favoreciendo la comunicación y la interacción entre un grupo de personas en la búsqueda de objetivos que les son comunes. Muchos autores han definido el Aprendizaje Colaborativo, entre ellos, Ariza y Oliva (2000): "la adquisición por individuos de conocimientos, habilidades o actitudes como resultado de la interacción grupal o, más brevemente, aprendizaje individual como resultado de un proceso grupal”.

Los foros son elementos esenciales de comunicación que permiten construir un aprendizaje compartido. Como afirma Benítez, "el foro de discusión en línea permite compartir entre todos los participantes sus reflexiones, búsquedas y hallazgos, así 
como establecer nexos directos entre dos o más integrantes con base en sus núcleos de interés y ámbitos de trabajo docente. En el foro la intervención de los asesores tiene como propósito incentivar el diálogo, conducir la discusión, realizar cierre de los debates y proponer líneas complementarias de conversación” (Benítez, 1999, p. 37). El aprendizaje colaborativo tiene sus fundamentos en la teoría de Vigotski sobre el Aprendizaje Social y está asociado a la teoría Social-Constructivista, esto implica estrategias de enseñanza y de evaluación que propicien en los estudiantes el desarrollo de un aprendizaje consciente y verdaderamente significativo (Brito, 2004).

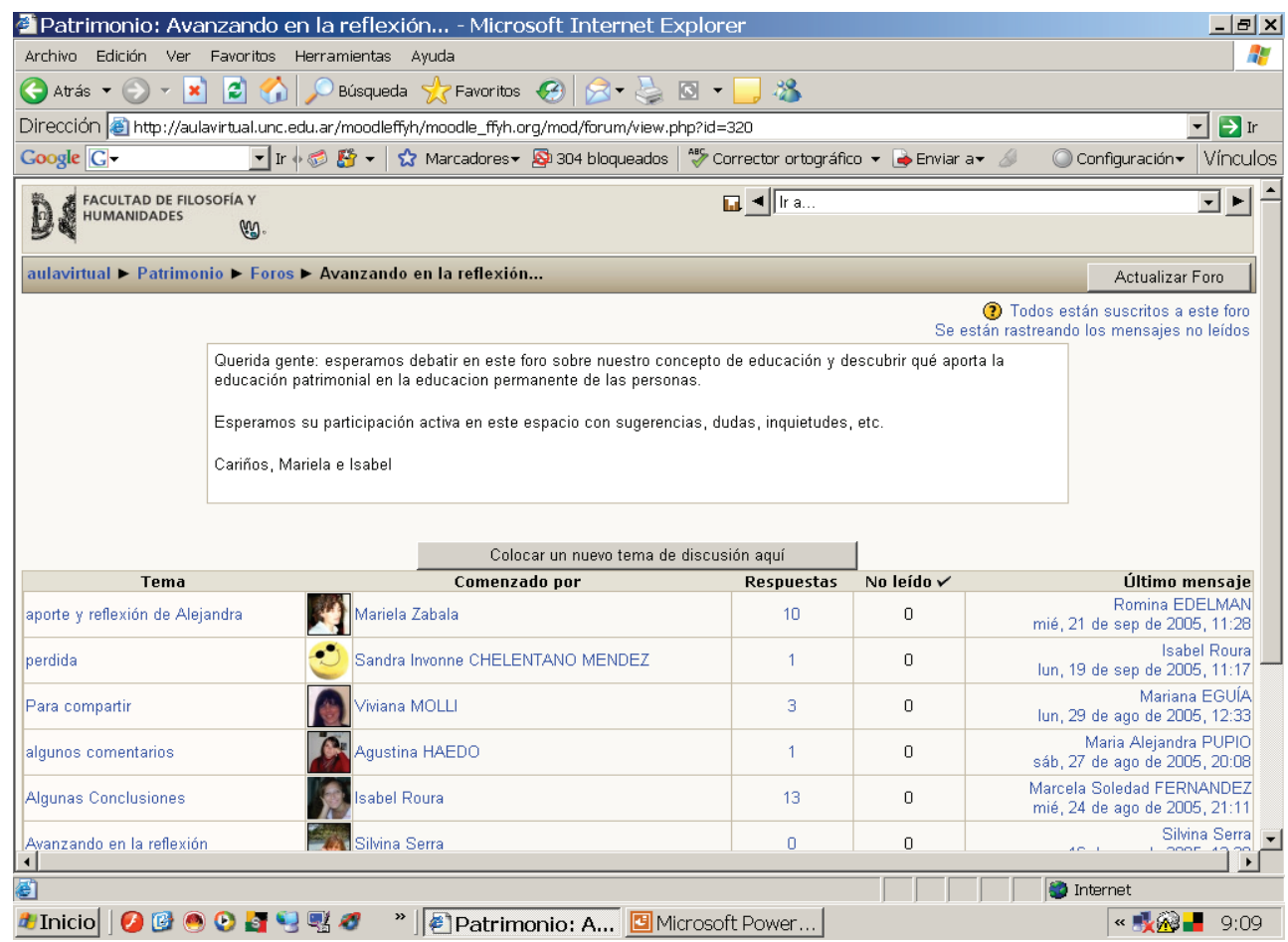

Imagen 1. Página de inicio por parte de las docentes de un foro para que el grupo clase se conozca en el curso de Patrimonio Arqueológico

Dentro de esta metodología de aprendizaje, también se trabaja con los alumnos a partir de consignas que deben resolver individualmente, haciendo especial hincapié en el desarrollo de procesos significativos que les permitan vincular su bagaje de conocimientos y capacidades con los nuevos contenidos. Valorando la complejidad de los procesos de aprendizaje y actuando desde esta perspectiva constructivista, el trabajo de la educación a distancia debe y puede atender no sólo a impartir nuevos 
conocimientos, sino también a ayudar a alumnos y alumnas a reorganizar su trama cognitiva y afectivo-valorativa con nuevos factores que se relacionen con lo que ellos y ellas ya sienten y conocen.

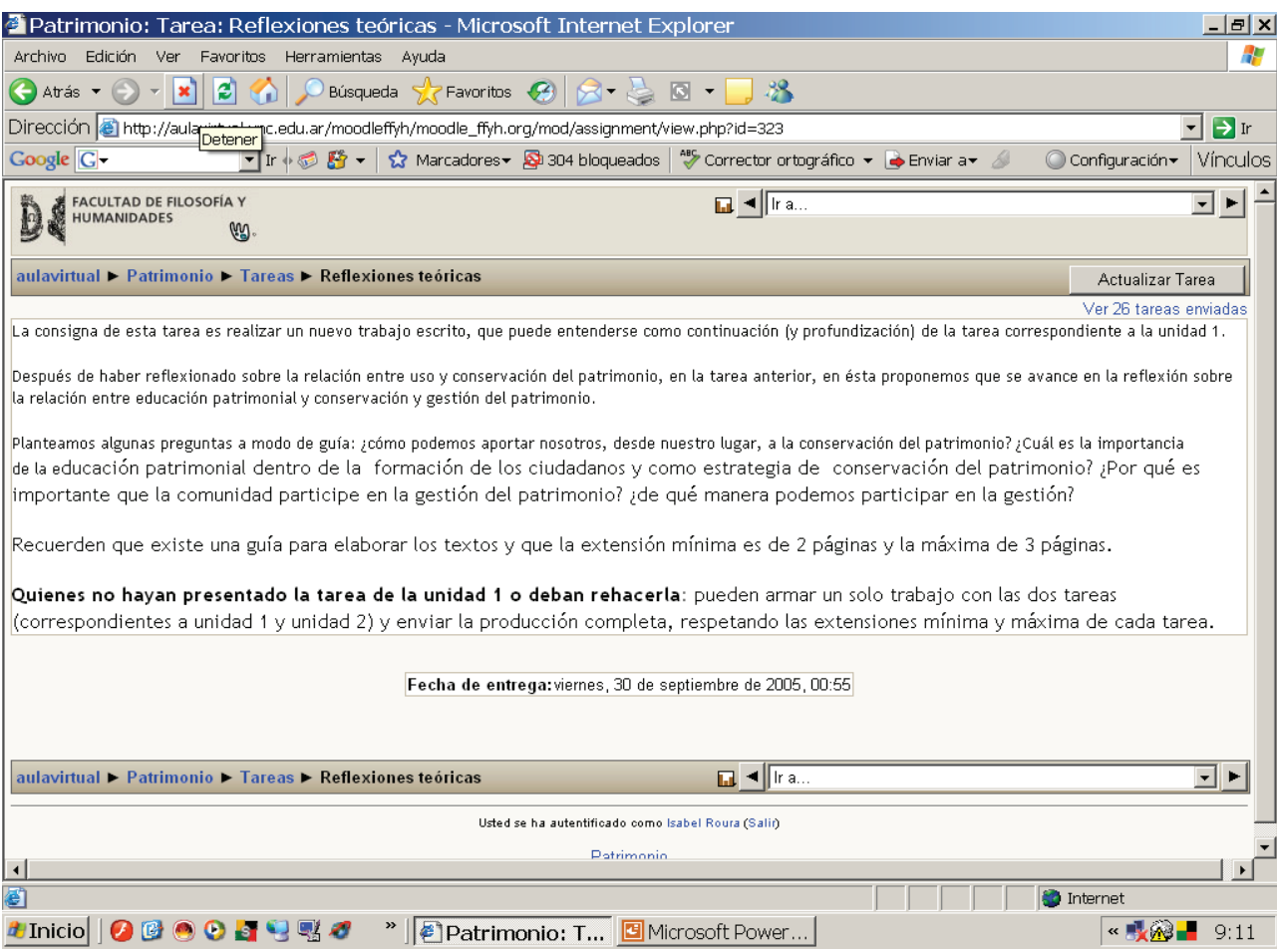

Imagen 2. Página donde se entregan las consignas de trabajo a los alumnos en el Curso de Patrimonio Arqueológico

El docente brinda las fuentes de información, despeja las dudas por medio de las tutorías, contesta a los requerimientos particulares de cada participante, etc., con un modelo educativo en el que lo fundamental no es enseñar conocimientos sino enseñar a aprender. La plasticidad de tal modelo queda así estrechamente vinculada a la función tutorial, ejercida de manera muy individualizada, para ayudar a cada persona en sus dudas o dificultades, lo que permite relaciones docente-alumno mucho más personalizadas que en los programas de capacitación presenciales. 

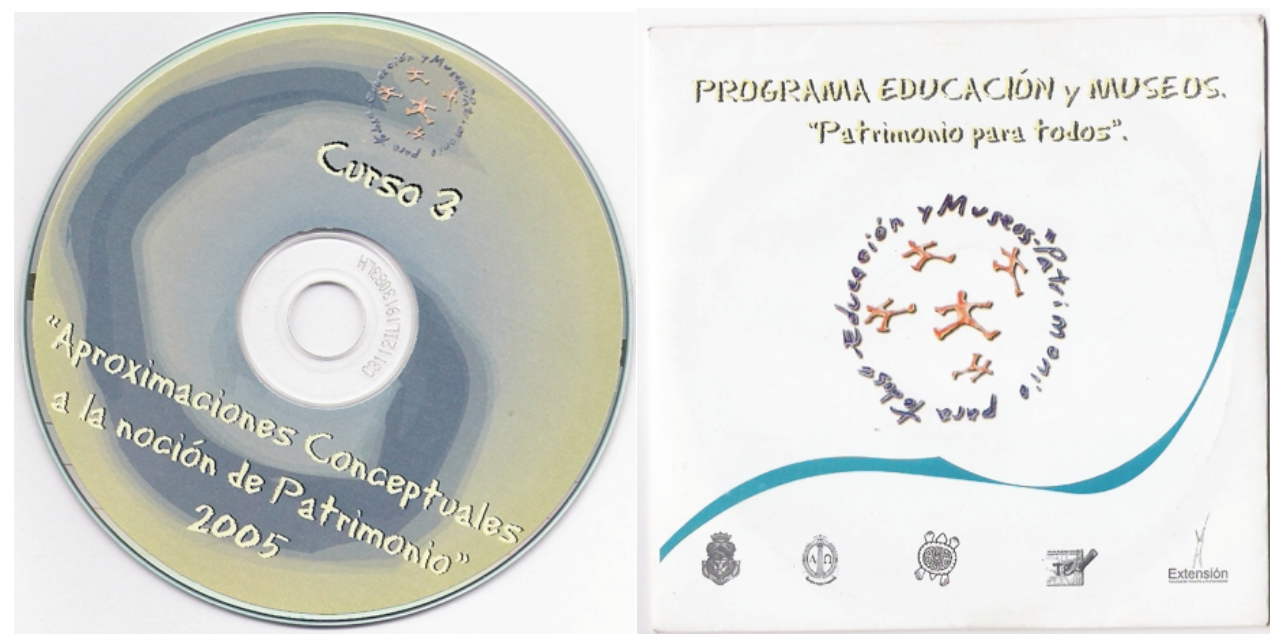

Imagen 3. Material de estudio del curso Aproximaciones Conceptúales a la Noción de Patrimonio, enviado a los alumnos por correo postal. El material bibliográfico se editó en un CD ROM.

En el caso del Curso Patrimonio Arqueológico la bibliografía se digitalizó y se colgó en la página.

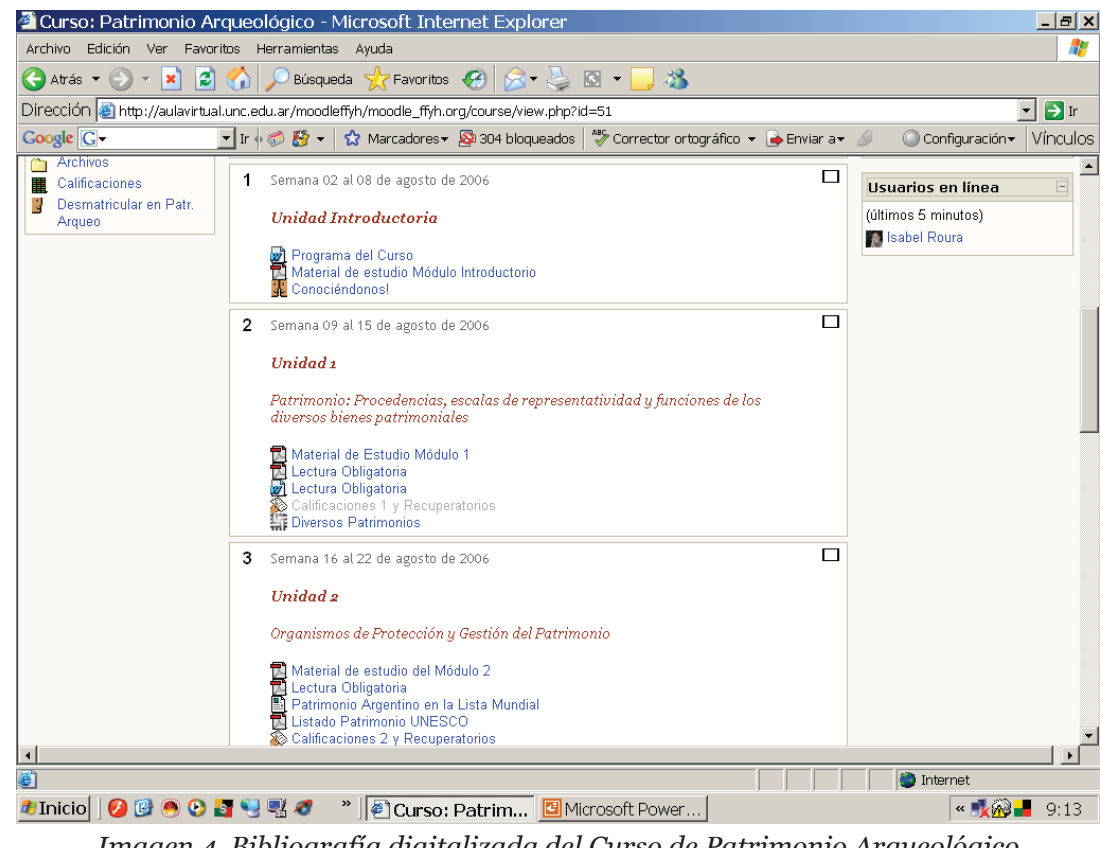


Dentro de los cursos dictados en el Programa "Educación y Museos. Patrimonio para todos", nuestra experiencia se limitó a dos de ellos: "Aproximaciones conceptuales a la noción de patrimonio" y "Patrimonio Arqueológico"

"Aproximaciones conceptuales a la noción de patrimonio" se dictó durante los años 2005 y 2007, con una modalidad virtual pero que contemplaba, además, dos encuentros presenciales.

La presentación del curso incluía un mapa conceptual con el fin de guiar, de una manera clara y precisa, los conceptos que serían abordados. Asimismo se contaba con un glosario donde se significaban los conceptos secundarios que también serían construidos o reconstruidos en el curso.

El programa de estudio de este curso se dividía en tres unidades de aprendizaje que articulan diferentes temas. Las unidades eran: 1- Patrimonio; 2- Educación patrimonial: el museo como escenario; y 3 - Otros escenarios posibles para la educación patrimonial. Cada unidad esta conformada por una presentación de la misma, un documento de trabajo elaborado por los docentes, dos o tres lecturas obligatorias y una última sección con el relato de experiencias. En el apartado de la bibliografía existía otra sección con el subtítulo “¿quién es quien?” En la que se presentaban a los autores con el fin de conocer desde donde y en que momento histórico está produciendo conocimiento ese investigador.

Las actividades que se proponían eran quincenales y por unidad. En cada actividad se sugerían pautas de lecturas, preguntas y se formulaba la consigna de trabajo práctico que se entregaba a los 15 días. Las actividades constaban de una parte de reflexión del tema a partir de la bibliografía propuesta y un análisis y consideración sobre la realidad en la que trabajaba cada alumno. Se buscaba que este trabajo fuera integrador.

La duración del curso era de un mes y quince días. Los que debían recuperar trabajos tenían quince días más después de finalizado el curso.

El primer dictado del curso se hizo en el año (2006- 2007). Algunos datos cualitativos sobre la evolución de la matrícula: se inscribieron al programa 87 alumnos de todo el país y 3 personas del extranjero. Participaron activamente en los debates, tareas de lectura y presentaciones 63. Para este curso se editaron CD Rom pero para la próxima edición (2007- 2008) se decidió colgar todo en la página web ya que se evaluaron algunas dificultades del trabajo con los CD Rom: problemas de 
correo postal; poseer una computador con Windows 2000; fallos en la grabación de los CD Rom.

En el desarrollo del curso para el 2007 se contó con 22 alumnos y todos de Argentina. Los encuentros presenciales fueron reemplazados, por razones de costos económicos, por un trabajo integrador que consistía en vivenciar un espacio patrimonial elegido por el alumno y hacer una evaluación crítica del mismo.

En ambos dictados la participación de los alumnos fue muy activa en los foros y en las distintas instancias del curso, generándose un rico intercambio de saberes y experiencias. El trabajo final logró un compromiso de los alumnos con su realidad patrimonial, a partir de los nuevos conocimientos construidos en este curso.

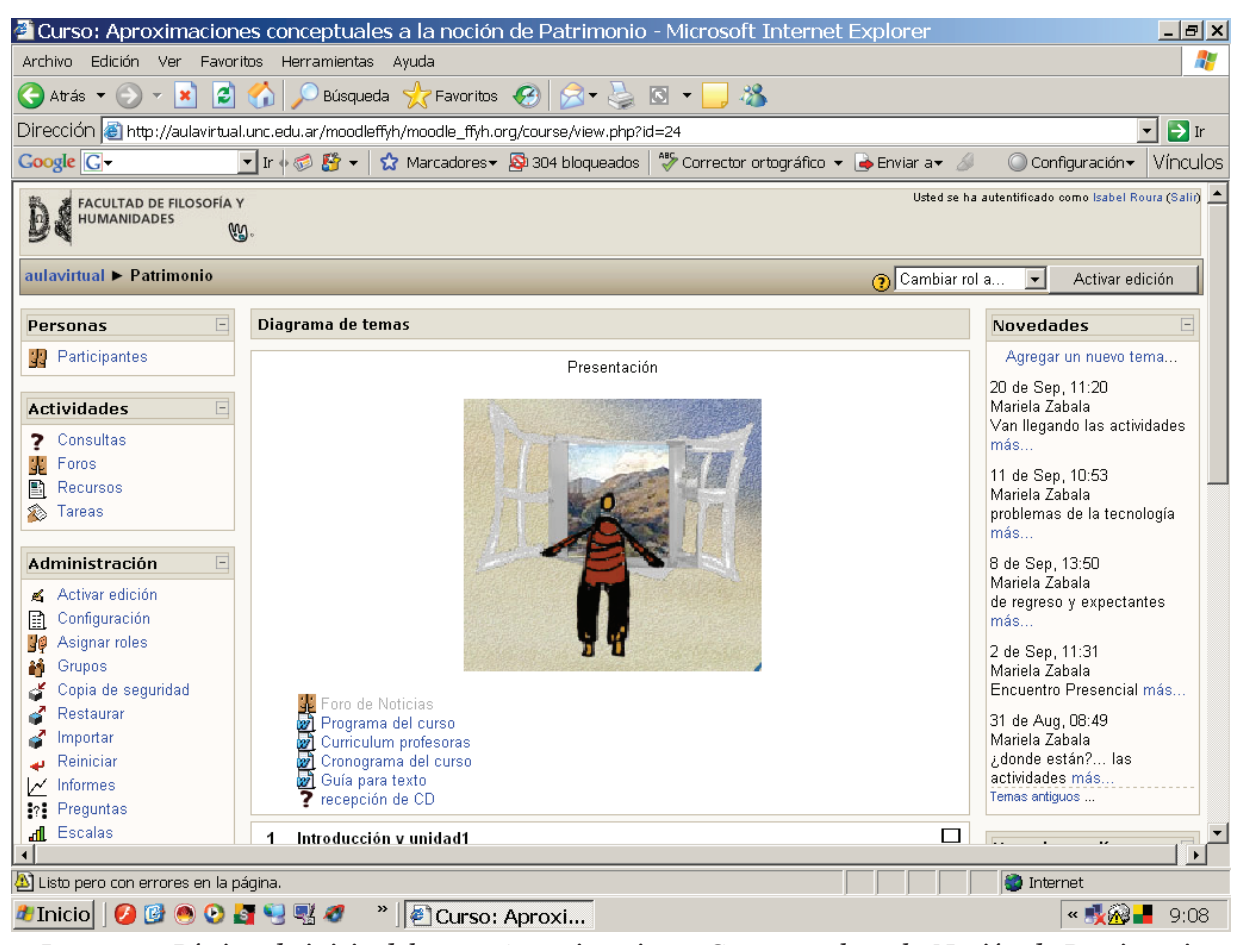

Imagen 5. Página de inicio del curso Aproximaciones Conceptuales a la Noción de Patrimonio

"Patrimonio Arqueológico" se caracterizó por ser un curso intensivo realizado durante dos meses, en dos años consecutivos, 2006 y 2007 y se desarrolló completamente on line. La periodicidad fue semanal, las clases se presentaban en el aula virtual junto con la actividad a desarrollar por los alumnos y la bibliografía (Assandri y Roura, 2006). 
El programa de estudio se articula mediante seis unidades de aprendizaje: Patrimonio, procedencias, escalas de representación y función de los diversos bienes patrimoniales, Organismos de protección y gestión del Patrimonio, Patrimonio Histórico y Arqueológico, Patrimonio Arqueológico: investigación y conservación, Patrimonio Arqueológico: gestión y difusión, y Patrimonio Arqueológico: legislación $\mathrm{y}$ un trabajo final integrador.

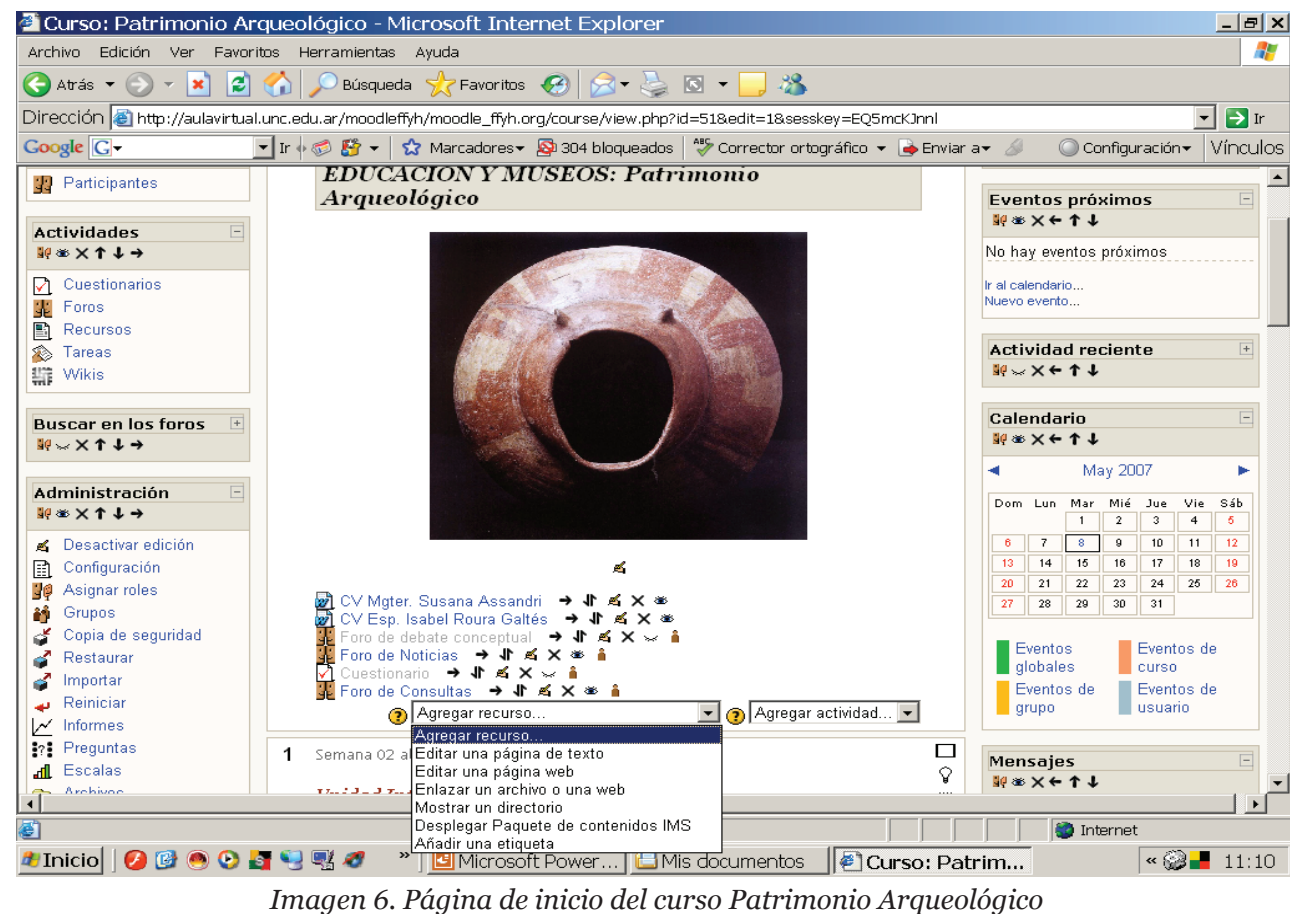

La comunidad virtual se integró con 2 profesores, 1 tutor, 1 apoyo administrativo, 1 integrante de la Comisión Académica, 1 coordinador y 43 alumnos en el año 2006, y 27 en el año siguiente.

En el primer año los alumnos provenían en su mayoría de la provincia de Córdoba, 26, 16 eran de otras provincias argentinas y 1 colombiano, de los cuales 34 fueron aprobados.

Durante el año 2007 los inscritos se integraron con 4 extranjeros (2 españoles, 1 venezolano y 1 guatemalteco) 13 de distintas provincias de Argentina y 6 de la provincia de Córdoba. El total de aprobados fueron 20. 
Ambos períodos se caracterizaron por la calidad en el rendimiento de los alumnos, tanto por su participación, como por el nivel de producción.

\section{ALGUNAS CONCLUSIONES A MODO DE CIERRE}

Si bien las propuestas fueron planificadas bajo planteamientos conceptuales sólidos, recién después de su puesta en marcha, evaluación y reflexión del proceso enseñanza aprendizaje es posible afirmar que los conceptos previos que guían el proyecto son factibles de abordar en una propuesta de capacitación en Internet.

Entre las ventajas de este proceso de aprendizaje, la más relevante es su capacidad de conformar una comunidad virtual con relaciones horizontales entre los participantes y no sólo establecer vínculos direccionales docente-alumno.

La heterogeneidad de los grupos es un factor positivo ya que enriquece el intercambio intergeneracional, interregional y multicultural en la construcción de conocimientos. Esta modalidad de enseñanza llega a un público diverso en su formación e interesado en continuar capacitándose, y, fundamentalmente, un público que no aspira a un título universitario, sino que necesita de estos espacios para enriquecer sus saberes y poder aplicarlos a sus prácticas laborales. Como ocurre, por ejemplo, con los directores de museos de zonas alejadas de las grandes ciudades.

Debemos destacar la importancia del seguimiento realizado por los docentes, manteniendo una conexión continua dentro de la plataforma, conteniendo a los alumnos frente a sus dudas o miedos, o para ampliar las explicaciones sobre las consignas de los trabajos.

La modalidad a distancia, con sus múltiples recursos, favorece el contacto entre docentes y alumnos, alcanzándose una enseñanza personalizada.

Entre las dificultades que se detectan en todas las experiencias, cabe destacar la falta de capacitación de los alumnos en el uso de la tecnología multimedia lo que significa destinar un período previo de aprendizaje sobre la utilización de la plataforma informática para poder avanzar en la construcción de los conocimientos específicos del curso y monitorear el proceso de utilización de las TIC a lo largo de todo el ciclo para aprovechar sus potencialidades. Por ello es muy importante la relación docente alumno, como ya lo mencionáramos más arriba. 
Finalmente consideramos que de este modo la Universidad está creando espacios e instrumentos nuevos como una instancia superadora de la educación superior tradicional, a la vez que está cubriendo una necesidad de formación, y contribuyendo en la construcción de una comunidad de los trabajadores de la cultura a través de un programa interdisciplinario que aborda el problema de los museos y el patrimonio sin imponer pautas universales. Es tarea de la Universidad no mantenerse alejada de los debates sobre patrimonio y educación en museos, promoviendo y aportando a la problematización de estos temas e incorporando las distintas miradas locales y regionales.

\section{NOTAS}

1 La oferta educativa de este programa comenzó en el año 2004 y aún continúa.

2 Moodle es un sistema de gestión de cursos libre (course management system CMS) que ayuda a los educadores a crear comunidades de aprendizaje en línea. Fue creado por Martín Dougiamas, quien era el administrador de WebCT en la Universidad Tecnológica de Curtin, y se basó en las ideas del constructivismo en pedagogía que afirman que el conocimiento se construye en la mente del estudiante en lugar de ser transmitido sin cambios a partir de libros o enseñanzas y en el aprendizaje colaborativo. Un profesor que opera desde este punto de vista crea un ambiente centrado en el estudiante que le ayuda a construir ese conocimiento con base en sus habilidades y conocimientos propios en lugar de simplemente publicar y transmitir la información que se considera que los estudiantes deben conocer.

Datos extraídos del sitio web de Moodle: http://docs.moodle.org/es/

3 La Junta Ejecutiva de ICOM en su reunión del 2000 en París aceptó el establecimiento provisional de este comité por tres años para luego evaluarlo con el fin de establecerlo definitivamente. Aún no se conocen los resultados de esa experiencia.

4 Un (o una) wiki es un sitio web colaborativo que puede ser editado por varios usuarios. Los usuarios de una wiki pueden crear, editar, borrar o modificar el contenido de una página web, de una forma interactiva, fácil y rápida.

\section{REFERENCIAS BIBLIOGRÁFICAS}

Ariza, A.; Oliva, S. (2000). Las Nuevas Tecnologías de la Información y la Comunicación y una Propuesta para el Trabajo Colaborativo. V Congreso Iberoamericano de Informática Educativa Chile. [en línea] Disponible en: http://www.c5.cl/ieinvestiga/actas/ ribie2000/papers/405/index.htm [consulta 2008, 25 de marzo]

Assandri, S.; Roura Galtés, I.; Zabala, M.
(2007). Educación Patrimonial en Internet. $V$ Jornadas de Encuentro Interdisciplinario "Las Ciencias Sociales y Humanas en Córdoba”. Secretaría de Investigación Ciencia y Técnica, Facultad de Filosofía y Humanidades, Universidad Nacional de Córdoba, Córdoba. Argentina

Assandri, S.; Roura Galtés, I. (2006)."Educación y museos: 
Patrimonio Arqueológico”. Museo de Antropología y Área de Tecnología Educativa. Universidad Nacional de Córdoba. Argentina. E-Book.

Assandri, S.; Zabala, M. (2002). Documentación de la Colección Jorge von Hauenschild. Jornadas de Ciencias Sociales y Humanas de la Facultad de Filosofía y Humanidades. Universidad Nacional de Córdoba, Córdoba. Argentina

Benítez García, R. (1999). Pedagogía y comunicación en la renovación docente. Revista Tecnología y Comunicación Educativas. $\mathrm{N}^{\mathrm{o}}$ 30. jul/dic. México. (3237).

Bonnin, M. (2006). Informe de gestión. Museo de Antropología. Facultad de Filosofía y Humanidades. Universidad Nacional de Córdoba. Argentina.

Bonnin, M. (2007). Informe de gestión. Museo de Antropología. Facultad de
Filosofía y Humanidades. Universidad Nacional de Córdoba. Argentina.

Brito, V. (2004). El foro electrónico: una herramienta tecnológica para facilitar el aprendizaje colaborativo.Edutec. Revista Electrónica de Tecnología Educativa. $\mathrm{N}^{\mathrm{o}}$ 17. España. [en línea] Disponible en: http://www.uib.es/depart/gte/ edutec-e/revelec17/brito_16a.htm [consulta 2008, 25 de marzo]

Roura Galtes, I.; Zabala, M. (2005). Aproximaciones conceptuales a la Noción de Patrimonio. CD Rom, 1a ED. Museo de Antropología y Área de Tecnología Educativa. Universidad Nacional de Córdoba. Argentina.

Zabala, M,; Roura Galtes, I. (2007) Aproximaciones conceptuales a la Noción de Patrimonio, $2^{\text {a }}$ ED. Museo de Antropología y Área de Tecnología Educativa. Universidad Nacional de Córdoba. Argentina.

\section{PERFIL ACADÉMICO Y PROFESIONAL DE LOS AUTORES}

Mariela Eleonora Zabala. Licenciada y Profesora en Historia. Maestrando en Antropología. Con capacitación en Estancia en Documentación de colecciones museológicas por el Ministerio de Educación, Cultura y Deporte de España. Coordinadora del Área Educación y Difusión del Museo de Antropología, Facultad de Filosofía y Humanidades, Universidad Nacional de Córdoba, Argentina. Profesora Adjunta en Museo de Antropología, Facultad de Filosofía y Humanidades, Universidad Nacional de Córdoba, Argentina. Docente de los programas: "Educación y Museos" y "Patrimonio Local y Sociedad", ambos de la Facultad de Filosofía y Humanidades, Universidad Nacional de Córdoba, Argentina.

E-mail: marielaeleonora@gmail.com

DIRECCION DE LA AUTORA:

Obispo Trejo 11732 "b".

Nueva Córdoba, Córdoba, Argentina.

(Código Postal 5000) 
María Isabel Roura Galtés. Arquitecta. Especialista en Educación Ambiental. Con capacitación de postgrado en Planificación del Paisaje y en Recuperación del Patrimonio Cultural y Natural. Adscripta al Área Educación y Difusión en Museo de Antropología, Facultad de Filosofía y Humanidades, Universidad Nacional de Córdoba, Argentina. Docente de los programas: "Educación y Museos" y "Patrimonio Local y Sociedad", ambos de la Facultad de Filosofía y Humanidades, Universidad Nacional de Córdoba, Argentina.

E-mail: isabel_roura@arnet.com.ar

DIRECCION DE LA AUTORA:

Copiapó 154, Córdoba, Argentina.

(Código Postal 5004)

Susana Beatriz Assandri. Licenciada en Historia. Facultad de Filosofía y Humanidades. Universidad Nacional de Córdoba. Argentina. Master en Arqueología Social de Iberoamérica. Universidad Internacional de Andalucía. España. Profesora Adjunta en Museo de Antropología, Facultad de Filosofíay Humanidades, Universidad Nacional de Córdoba, Argentina. Docente de los programas: "Educación y Museos" y "Patrimonio Local y Sociedad", ambos de la Facultad de Filosofía y Humanidades, Universidad Nacional de Córdoba, Argentina.

E-mail: suassandri@gmail.com

DIRECCION DE LA AUTORA:

Victorino Rodríguez 1859,

Córdoba, Argentina.

(Código Postal 5009)

Fecha de recepción del artículo: 17/06/09

Fecha de aceptación del artículo: 26/01/10 